\title{
Oscillatory and Vanishing Resistance States in Microwave Irradiated 2D Electron Systems
}

\author{
R. R. Du*, M. A. Zudov ${ }^{\dagger}$, M. A. Zudov, C. L. Yang, and Z. Q. Yuan \\ Department of Physics, University of Utah, Salt Lake City, Utah 84112, USA \\ L. N. Pfeiffer and K. W. West \\ Bell Laboratories, Lucent Technologies, New Jersey 07974, USA
}

\begin{abstract}
Giant-amplitude oscillations in dc magnetoresistance of a high-mobility two-dimensional electron system can be induced by millimeterwave irradiations, leading to zero-resistance states at the oscillation minima. Following a brief overview of the now well-known phenomenon, this paper reports on aspects of more recent experiments on the subject. These are: new zero-resistance states associated with multi-photon processes; suppression of Shubnikov-de Haas oscillations by high-frequency microwaves; and microwave photoconductivity of a high-mobility two-dimensional hole system.
\end{abstract}

Keywords: Two-dimensional electronic system; Magnetotransport; Microwave

* Corresponding author: rrd@physics.utah.edu

$\dagger$ Present address: School of Physics and Astronomy, University of Minnesota, Minneapolis, MN 55455, USA 


\section{INTRODUCTION}

Under irradiation of microwaves dramatic new effects occur in two-dimensional electron systems (2DES) in GaAs-AlGaAs heterostructures previously used to study quantum Hall effects (QHE) $\stackrel{1}{2}$ As first reported by Zudov et al.,$^{2} \frac{2}{2}$ and also by Ye et al. $\frac{3}{\underline{2}}$ in the millimeterwave (MW) frequency range $(f=30-150 \mathrm{GHz})$ and in a small magnetic field $(B<0.5 \mathrm{~T})$, at low temperatures $(T \sim 1 \mathrm{~K})$ a new type of oscillations arises in the magnetoresistance $R_{x x}$ of a high-mobility 2DES. These oscillations are periodic in $1 / B$ and can occur in a $B$ weaker than the onset of Shubinikov-de Haas oscillations ( $\mathrm{SdH})$. Characteristically, their period is controlled by the ratio of the microwave frequency to the electron cyclotron frequency, $\epsilon=\omega / \omega_{c}$, where $\omega_{c}=e B / m^{*}, m^{*}$ is the effective mass of the conduction band electrons in GaAs. In particular, the oscillations can be associated with cyclotron resonance (CR, $\epsilon=1)$ and its harmonics $(\epsilon=2,3,4, \ldots)$. Although the photoconductivity (PC) effect was well-known in the literature ${ }^{4.5 .6}$ such findings came as a surprise. Not only the observation of higher-order CR (hence oscillations rather than a single peak), but also its large amplitude, was completely unexpected. Moreover, later it was found that in samples of even higher mobility, the oscillation minima approach zero. Recently, Mani et al $\underline{7}^{7}$ and Zudov et al. ${ }^{8}$ reported the observation of new "zero-resistance states" (ZRS) associated with such minima in very clean samples. At low temperatures, ZRS are characterized by an exponentially vanishing diagonal resistance and an essentially classical Hall resistance. Such a new effect in a 2DES induced by microwaves is a subject of much current experimental 19.10.11.12.13.14 and theoretical work. $15,16,17,18,19,20,21$ Some of the theoretical ideas can be traced back to earlier studies of negative photoconductivity phenomena in semiconductors. ${ }^{22}$ These models, as well as others, remain to be tested experimentally. The observation of ZRS is complemented by an observation of zero-conductance states under similar experimental conditions, but in Corbino samples. ${ }^{9}$

\section{OVERVIEW OF THE EFFECT}

Photoconductivity related to CR of a 2DES was first reported by Maan et $a l . \underline{4}$ in the farinfrared frequency range and in a strong magnetic field. In the MW frequency range, distinct magnetoresistance signals due to electron spin resonance (Dobers et al..$^{\frac{5}{}}$ ) or magnetoplasmon 
resonance (Vasiliaou et al. ${ }^{6}$ ) were observed in 2DES in GaAs-AlGaAs heterostructures. In these earlier studies using samples of electron mobility $\mu \sim 1 \times 10^{6} \mathrm{~cm}^{2} / \mathrm{Vs}$ or less, the PC signal exhibits a single peak in the $R_{x x}$, and its amplitude is typically less than $1 \%$. The MW-induced giant amplitude magnetorsistance oscillations were discovered ${ }^{2}$ using a highmobility $\left(3 \times 10^{6} \mathrm{~cm}^{2} / \mathrm{Vs}\right)$ 2DES. In a small magnetic field the $R_{x x}$ shows periodic (in $1 / B$ ) oscillations, with the $B$ positions of major maxima and minima in the oscillatory structure roughly conforming to $\frac{12}{2}$

$$
\omega / \omega_{c}=\epsilon=\left\{\begin{array}{ll}
j & \text { maxima } \\
j+1 / 2 & \text { minima }
\end{array} \quad j=1,2,3, \ldots\right.
$$

Phenomenologically such oscillations resemble SdH oscillations except that their period relates to $\epsilon$ rather than to $\nu$, the Landau level filling factor. Most remarkably, the $R_{x x}$ at the minima was found to reduce from its dark value, indicating of negative PC. This was a crucial step leading to the discovery of ZRS; increasing sample mobility favors oscillation amplitude, and, therefore, the $R_{x x}$ value at the minima becomes progressively smaller under similar experimental conditions.

Vanishingly small $R_{x x}$ at the minima was initially observed in a GaAs/ $\mathrm{Al}_{0.3} \mathrm{Ga}_{0.7} \mathrm{As}$ heterojunction 2DES approaching a mobility $\mu=10 \times 10^{6} \mathrm{~cm}^{2} / \mathrm{Vs}^{23}$ The data of Yang et al. are shown in Fig. 1. As marked by arrows, in this sample the strongest minimum in the $R_{x x}$ evolves into ZRS as the MW frequency is increased (hence, the minimum moves towards higher $B)$.

Our current data from an ultraclean 2DES 8 are shown in Fig. 2. The sample was cleaved from a $\mathrm{Al}_{0.24} \mathrm{Ga}_{0.76} \mathrm{As} / \mathrm{GaAs} / \mathrm{Al}_{0.24} \mathrm{Ga}_{0.76} \mathrm{As} \mathrm{QW}$ (width $30 \mathrm{~nm}$ ), with a mobility $\mu=25 \times 10^{6}$ $\mathrm{cm}^{2} / \mathrm{Vs}$ and a density $n_{e}=3.5 \times 10^{11} \mathrm{~cm}^{-2}$, respectively. With the irradiation of MW $(f=57$ $\mathrm{GHz}$ and the power incident on the sample surface $P \sim 100 \mu \mathrm{W})$, the $R_{x x}$ shows a series of oscillations and the novel zero-resistance states at the first four minima.

The width of the ZRS encompasses a large range of the filling factor $\nu$. For example, the first ZRS spans from $\nu \sim 115$ to $\nu \sim 155$ and similar width of $\Delta \nu \sim 40$ is observed for other ZRS minima in this sample. These data indicate that the transport under MWs is controlled by $\epsilon$ rather than by $\nu$. While the $R_{x x}$ here remarkably resembles that in QHE, the Hall resistance, $R_{x y}$, shows no sign of forming plateaus. In the very-high mobility $2 \mathrm{DES}$, the major peaks of MW-induced oscillations (which are relevant to ZRS regime) can still be well described by Eq. 1 for $j=1,2,3$, but their shapes are largely asymmetric. Higher-order 


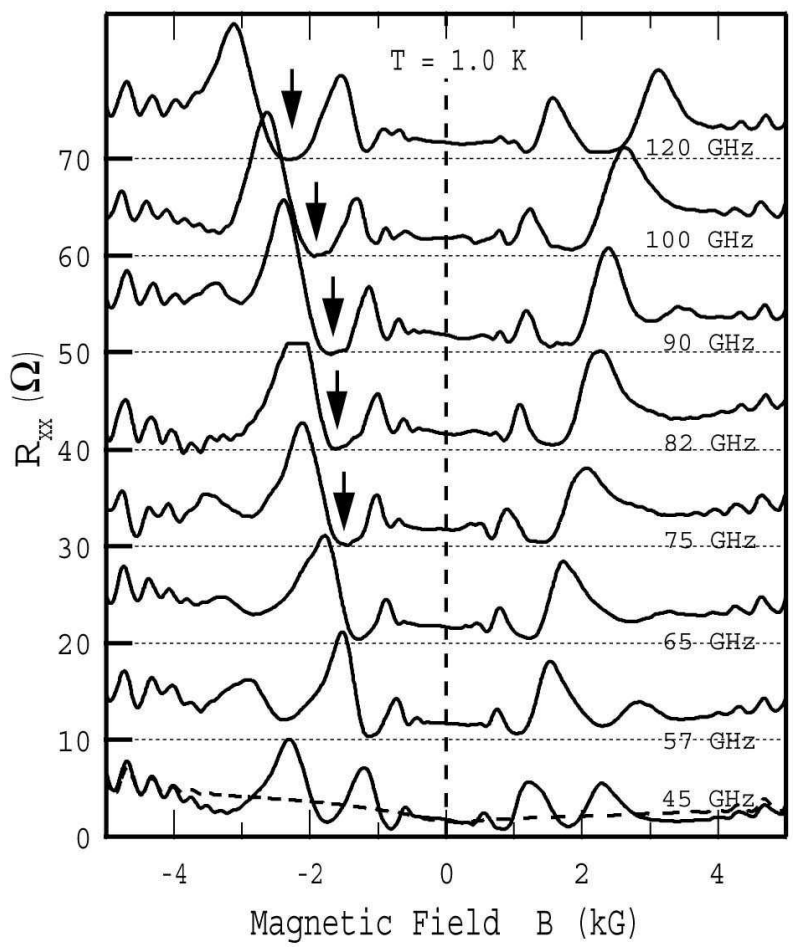

FIG. 1: Microwave induced oscillation minimum evolves into zero-resistance state as the microwave frequency is increased. The sample is a GaAs/ $\mathrm{Al}_{0.3} \mathrm{Ga}_{0.7} \mathrm{As}$ heterojunction, having a 2DES of density $n_{e}=2.7 \times 10^{11} \mathrm{~cm}^{-2}$ and mobility $\mu=1 \times 10^{7} \mathrm{~cm}^{2} / \mathrm{Vs}$.

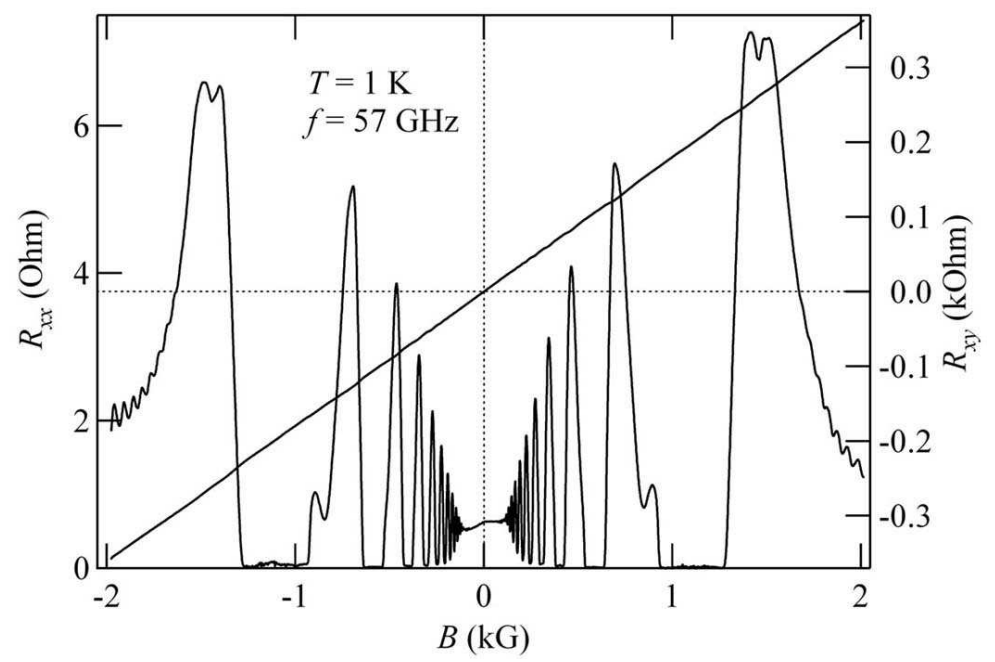

FIG. 2: Electrical transport data in a Hall sample of a very high mobility quantum well exposed to millimeter waves. The magnetoresistance $R_{x x}$ shows sharp oscillations and a series of zeroresistance states; Hall resistance $R_{x y}$ shows an essentially classical, linear dependence on B. 

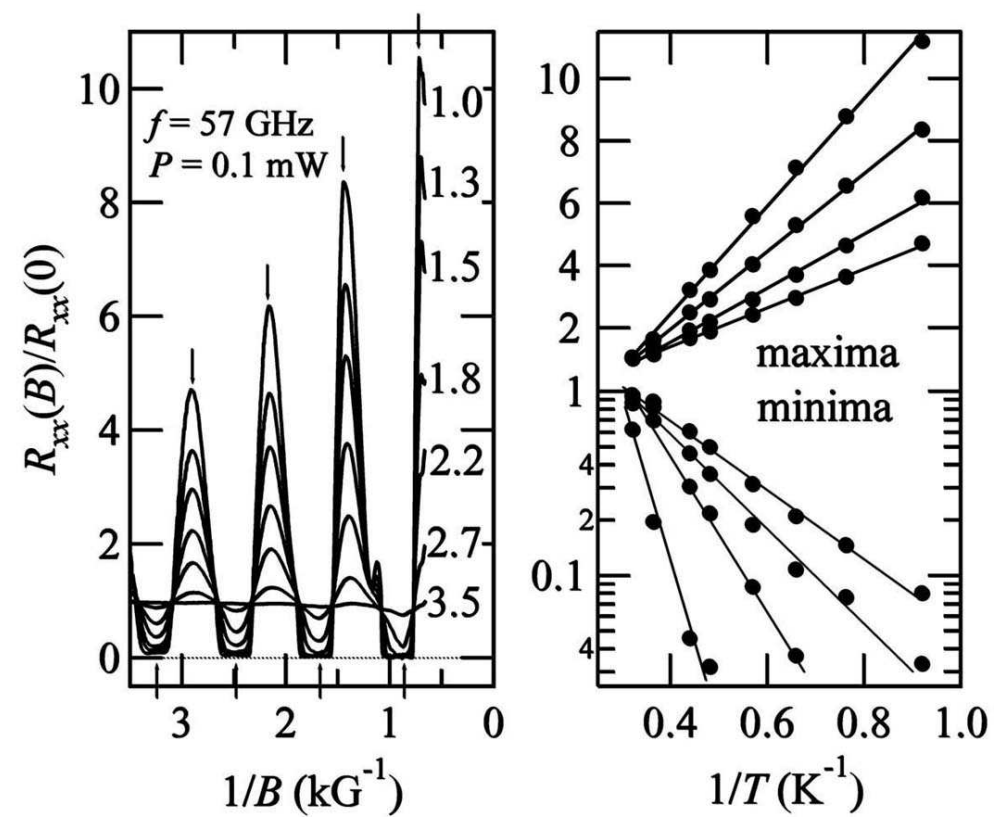

FIG. 3: The temperature dependence of $R_{x x}$ peaks and ZRS minima, showing a roughly linear dependence of the peak values on inverse temperature, and a thermally activated resistance at the minima.

peaks are better described by a $B$-dependent phase shift with respect to $\mathrm{CR}$ harmonics $(j=4,5, \ldots)$, which saturates at $-1 / 4 .^{12}$

One of the most interesting and puzzling results in the ZRS regime is the temperature dependence. The $1 / B$ oscillations are shown in Fig. 3 for different temperatures but under the same MW irradiation. Below a temperature $T \sim 4 \mathrm{~K}$ both the maxima and the minima exhibit strong temperature dependence. Using standard Arrhenius plot we present the $R_{x x}(B) / R_{x x}(0)$ of the first four minima on a logarithmic scale, versus $1 / T$. All four minima conform to a general expression, $R_{x x}(T) \sim \exp \left(-T_{0} / T\right)$, over at least one decade in the $R_{x x}$. Unusually large $T_{0}$ is found for these minima. For example, $T_{0} \approx 20 \mathrm{~K}$ for the first minimum, exceeds both the MW photon energy $(\sim 3 \mathrm{~K})$ and the Landau level spacing $(\sim 2$ $\mathrm{K}$ ) by about an order of magnitude. As also shown in Fig. 3, the $R_{x x}$ peaks are roughly linear with $1 / T$.

Using Corbino samples, we have observed zero-conductance states (ZCS) under similar experimental conditions. ${ }^{9}$ Typical data from a high mobility $\left(\mu \approx 12.8 \times 10^{11} \mathrm{~cm}^{2} / \mathrm{Vs}\right) 2 \mathrm{DEG}$ Corbino ring are shown in Fig. 4. Temperature dependence, as well as I-V measurements at ZCS, shows that 2DES behaves like a macroscopic insulator in this regime. ${ }^{9}$ 


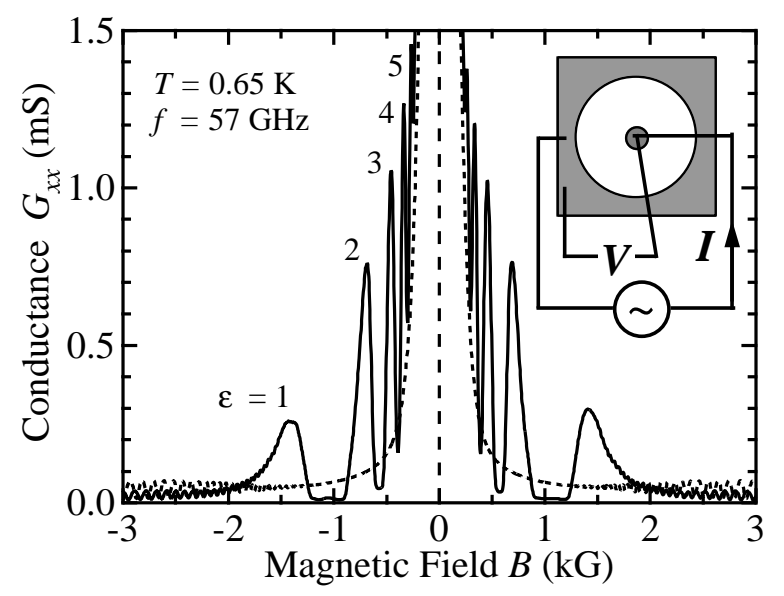

FIG. 4: Microwave-induced conductance oscillations and zero-conductance states in 2DES measured in a Corbino sample.

\section{MULTI-PHOTON PROCESSES}

Remarkably, we have observed a series of new ZRS associated with fractional $\epsilon$, such as $\epsilon=1 / 2$ and $3 / 2$, in an ultraclean 2DEG sample (similar to the sample used in Fig. 2) exposed to MW of lower frequency $(f<30 \mathrm{GHz})$. Figure 5 shows the development of such ZRS with increasing MW power $P$. At all $P$ values the previously reported "integer" ZRS are observed. Beyond the $\epsilon=1$ peak, a $\epsilon=1 / 2$ peak and associated minimum (around $B \sim 0.1$ T) are already visible at lower power (attenuation $-8 \mathrm{~dB}$ ). With increasing $P$, this minimum develops into a new wide ZRS. The $\epsilon=1 / 2$ structure has first been noticed in Ref. 2 and can also be seen in Fig. 1; the feature becomes stronger in samples with higher mobility $\frac{8}{*}$ Dorozhkin $^{10}$ and Willett et al. ${ }^{11}$ have also reported features associated with $\epsilon=1 / 2$. In this experiment we also observe fractional ZRS forming about $\epsilon=3 / 2$. Another important observation is the (overall) suppression of the $R_{x x}$ at $\epsilon<1 / 2$.

From the $B$ position we interpret these new ZRS as resulting from two-photon processes, where two photons of frequency $\omega$ participate in the transition between Landau levels separated by $\omega_{c}$ and $3 \omega_{c}$, respectively $\underline{\underline{12}}$. This observation may suggest the importance of virtual processes in the microwave-induced oscillations and ZRS.

In the higher frequency region $(>100 \mathrm{GHz})$, we observed a strong modulation of SdH oscillations by the presence of MW. This phenomenon has been reported in Ref. 13 for samples of a moderately-high mobility. The data in Fig. 6 shows similar results from an 


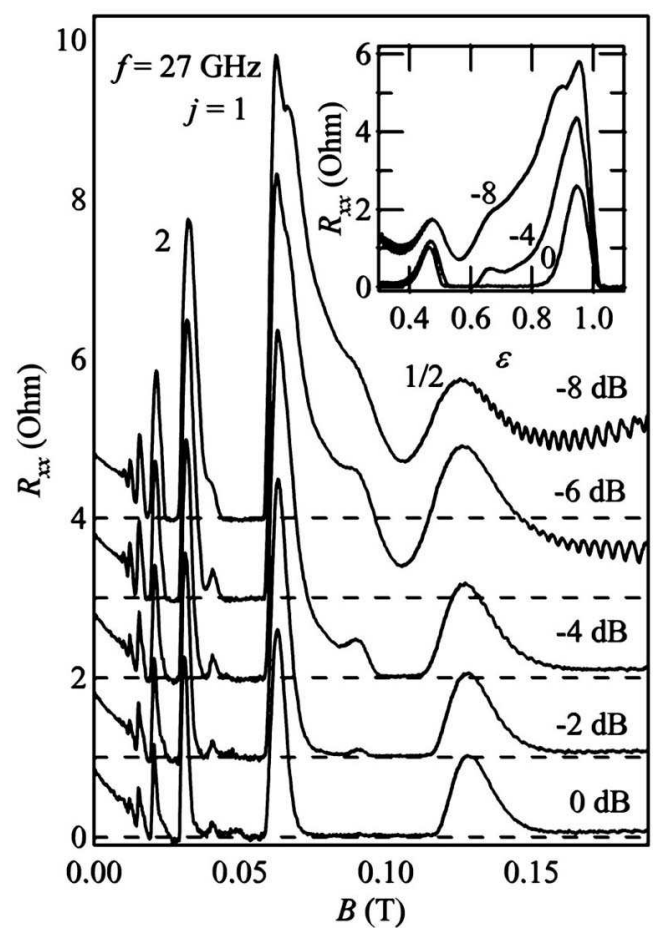

FIG. 5: Formation of zero-resistance states associated with transitions promoted by two microwave photons is shown. Maxima appear near $\epsilon=1 / 2$ and $\epsilon=3 / 2$; the associated minima develop into ZRS as the microwave power is increased (attenuation of the microwave power is marked next to the $R_{x x}$ traces).
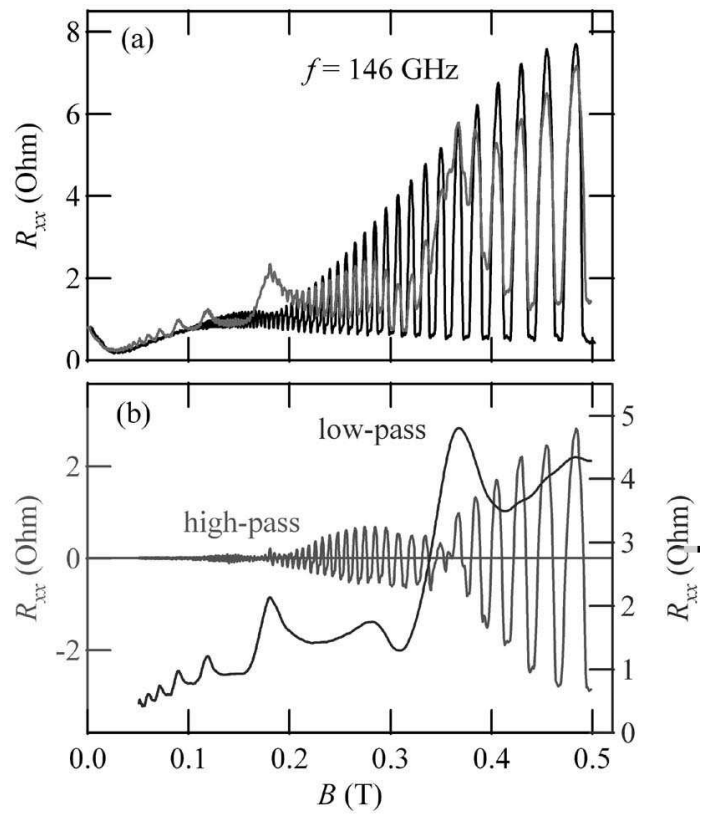

FIG. 6: The Shubinikov de-Haas oscillations are modulated by microwave-induced oscillations. 
ultra-clean 2DES. In addition to the first node, higher-order nodes were observed in this sample.

\section{PHOTOCONDUCTIVITY OF A 2D HOLE SYSTEM}

It is interesting to investigate the photoconductivity oscillations and ZRS in a highmobility 2D hole system (2DHS) primarily for the following reasons: 1) comparing with 2D electron system, 2DHS has different band structure parameters such as larger effective mass and significant nonparabolicity of the dispersion; 2) 2DHS possesses strong spin-orbit interactions. Our preliminary result for a 2DHS is shown in Fig. 7. The 2DHS is from a (001) GaAs $/ \mathrm{Al}_{0.4} \mathrm{Ga}_{0.6} \mathrm{As} \mathrm{QW}$ grown by MBE. The QW has a well width of $15 \mathrm{~nm}$; holes are provided by a $\mathrm{C}$ delta-doping layer situated $50 \mathrm{~nm}$ away from the well. A 100- $\mu \mathrm{m}$-wide Hall bar, on which measurements were performed, is defined by lithography and is contacted by In/Zn alloy.

To simultaneously detect photoresistivity, $\Delta \rho_{x x}=\rho_{x x}^{M W}-\rho_{x x}^{D A R K}$, and resistivity, $\rho_{x x}$, a double-modulation technique was employed. The main feature of $\Delta \rho_{x x}$, as shown for MW frequency of $26.5 \mathrm{GHz}$, is a positive peak at $B \approx 2.5 \mathrm{kG}$ and a negative valley (a suppression of $\rho_{x x}$ ) around $\sim 6 \mathrm{kG}$, both having relatively small magnitude. The $2.5 \mathrm{kG}$ peak could be

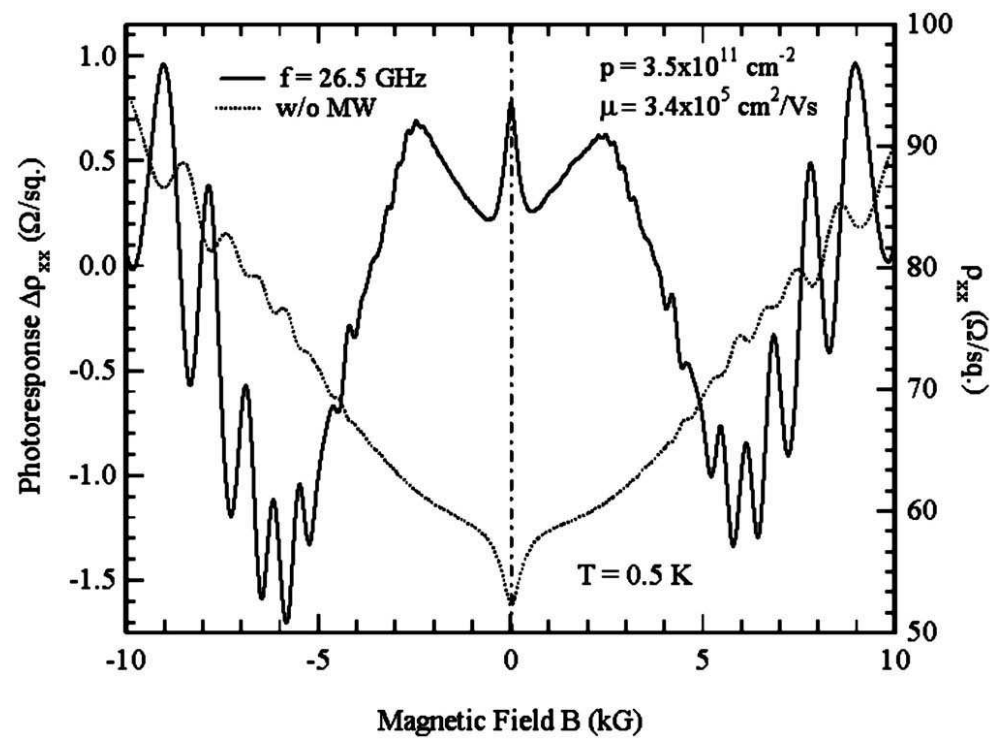

FIG. 7: A high-mobility 2D hole system in GaAs/AlGaAs quantum well shows sharp positive peak and negative valley in photoresistance $\Delta \rho_{x x}$ under microwave irradiation. 
associated with the $\mathrm{CR}$ of the $2 \mathrm{D}$ holes which have an effective mass $m^{*} \sim 0.3 m_{e}$ (measured in separate transmission experiments in a similar $B$ range; for measurement techniques, see Ref. 24). The nature of the $6 \mathrm{kG}$ valley is not yet clear.

From the mobility of the holes, $\mu=3.4 \times 10^{5} \mathrm{~cm}^{2} / \mathrm{Vs}$ and a mass value of $0.3 \mathrm{~m}_{e}$, we estimate a transport scattering time of $\tau_{t}=\mu m^{*} / e \approx 58 \mathrm{ps}$. This value is a factor of two smaller than that of the 2DES (where $\tau_{t} \approx 115 \mathrm{ps)} \mathrm{in} \mathrm{which} \mathrm{the} \mathrm{giant} \mathrm{amplitude} \mathrm{oscillations}$ were originally observed, $\frac{2}{2}$ a fact that may be considered in explaining the lack of oscillations in this 2DHS. However, the band structure of holes must be taken into account to understand these data.

\section{SUMMARY}

Oscillatory and vanishing magnetoresistance can be induced by millimeterwave irradiation in high mobility two-dimensional electronic systems traditionally used for studies of the quantum Hall effect. Depending on the sample geometry, zero resistance states (in Hall samples) or zero conductance states (in Corbino samples) are observed. Some new aspects of the observations are reported here which may help to distinguish between competing theoretical models of the phenomenon.

\section{Acknowledgments}

This work was supported by NSF, by DARPA, and by A. P. Sloan Foundation.

1 Perspective in Quantum Hall Effects, Ed. S. Das Sarma and A. Pinczuk (Wiley, New York, 1996).

2 M.A. Zudov, R.R. Du, J.A. Simmons, and J.L. Reno, Phys. Rev. B 64, 201311(R) (2001); see also cond-mat/9711149 (1997).

3 P.D. Ye, L.W. Engel, D.C. Tsui, J.A. Simmons, J.R. Wendt, G.A. Vawter, and J.L. Reno, Appl. Phys. Lett. 79, 2193 (2001).

4 J.C. Maan, Th. Englert, and D.C. Tsui, Appl. Phys. Lett. 40, 609 (1982).

5 M. Dobers, K. v. Klitzing, and G. Weimann, Phys. Rev. B38, 5453 (1988). 
6 E. Vasiliadou, G. Muller, D. Heitmann, D. Weiss, K. v. Klitzing, H. Nickel, W. Schlapp, and R. Losch, Phys. Rev. B 48, 17145 (1993).

7 R.G. Mani, J.H. Smet, K. von Klitzing, V. Narayanamurti, W.B. Johnson, and V. Umansky, Nature 420, 646 (2002); Phys. Rev. Lett. 92, 146801 (2004); Phys. Rev. B 69, 161306 (2004).

8 M.A. Zudov, R.R. Du, L.N. Pfeiffer, and K.W. West, Phys. Rev. Lett. 90, 046807 (2003).

9 C.L. Yang, M.A. Zudov, T.A. Knuuttila, R.R. Du, L.N. Pfeiffer, and K.W. West, Phys. Rev. Lett. 91, 096803 (2003).

10 S.I. Dorozhkin, JETP Lett. 77, 577 (2003).

11 R. G. Willett, L. N. Pfeiffer, and K. W. West, Phys. Rev. Lett. 93, 026804 (2004).

12 A detailed analysis of the oscillation phase can be found in M. A. Zudov, Phys. Rev. B 69, 041304(R) (2004).

13 A. E. Kovalev, S. A. Zvyagin, C. R. Bowers, J. L. Reno, and J. A. Simmons, Solid State Commun. 130, 379 (2004).

14 S. A. Studenikin, M. Potemski, A. Sachrajda, M. Hilke, L. N. Pfeiffer, and K. W. West, cond-mat/0404411

15 A.C. Durst, S. Sachdev, N. Read, and S.M. Girvin, Phys. Rev. Lett. 91, 086803 (2003).

16 P.W. Anderson and W.F. Brinkman, cond-mat/0302129.

17 A.V. Andreev, I.L. Aleiner, and A.J. Millis, Phys. Rev. Lett. 91, 056803 (2003).

18 I. A. Dmitriev, M. G. Vavilov, I. L. Aleiner, A. D. Mirlin, and D. G. Polyakov, cond-mat/0310668

19 I. A. Dmitriev, A. D. Mirlin, and D. G. Polyakov, Phys. Rev. Lett. 91, 226802 (2003).

20 M. G. Vavilov and I. L. Aleiner, Phys. Rev. B 69, 035303 (2004).

21 See, e.g., references in R. R. Du et al., Physica E22, 7 (2004).

22 V.I. Ryzhii, Sov. Phys. Solid State 11, 2078 (1970); V.I. Ryzhii, R.A. Suris, and B.S. Shchamkhalova, Sov. Phys. Semiconductors 20, 1289 (1986).

23 C. L. Yang, M. A. Zudov, R. R. Du, L. N. Pfeiffer and K. W. West, 2000, unpublished.

24 B. E. Cole, F. M. Peeters, A. Ardavan, S. O. Hill, J. Singleton, W. Batty, J. M. Chamberlain, A. Polisskii, M. Henini, and T. Cheng, J. Phys.: Condens. Matter 9, 3163 (1997). 